\title{
Downregulation of microRNA-3934-5p induces apoptosis and inhibits the proliferation of neuroblastoma cells by targeting TP53INP1
}

\author{
WEI YE ${ }^{1}$, FULV LIANG $^{2}$, CHEN YING $^{3}$, MAOLIN ZHANG $^{4}$, DONGBO FENG $^{5}$ and XINYU JIANG ${ }^{6}$
}

${ }^{1}$ Department of Neurology, Jianou Municipal Hospital, Jianou, Fujian 353100;

${ }^{2}$ Department of Urology, The Third Hospital of Xiamen, Xiamen, Fujian 361000; ${ }^{3}$ Department of Urology,

Haicang Hospital of Xiamen, Xiamen, Fujian 361026; ${ }^{4}$ Department of Surgery, Xiapu County Hospital, Xiapu County, Ningde, Fujian 355100; ${ }^{5}$ Department of Sports Medicine, The Central Hospital of Yongzhou, Yongzhou, Hunan 425000; ${ }^{6}$ Department of General Surgery, Xiamen Maternity and Child Health Care Hospital, Xiamen, Fujian 361000, P.R. China

Received August 18, 2018; Accepted March 21, 2019

DOI: $10.3892 /$ etm.2019.8007

\begin{abstract}
Neuroblastoma is the most common pediatric extracranial solid tumour in the world. miRNAs are a group of endogenous small non-coding RNAs that act on target genes to serve critical roles in many biological processes. Presently, the expression and role of miR-3934-5p in neuroblastoma remains unclear. Therefore, the aim of the present study was to investigate the expression of miR-3934-5p in neuroblastoma tissues and cell lines and to assess the role of miR-3934-5p in neuroblastoma. In the current study, the results revealed that miR-3934-5p was significantly upregulated in neuroblastoma tissues and cell lines. The data also identified TP53INP1 as a direct target gene of miR-3934-5p, which was negatively regulated by miR-3934-5p. The present study further demonstrated that TP53INP1 was downregulated in both neuroblastoma tissues and cell lines. Furthermore, the results of the current study indicate that miR-3934-5p downregulation may induce apoptosis and inhibit neuroblastoma cell viability. However, these effects were reversed via TP53INP1-siRNA. Data from the current study indicates that the miR-3934-5p/TP53INP1 axis may be a novel therapeutic target for neuroblastoma treatment.
\end{abstract}

\section{Introduction}

Neuroblastoma is a childhood cancer that affects $\sim 1 / 7,000$ children (1). Neuroblastoma is the most common pediatric extracranial solid tumor worldwide that originates

Correspondence to: Dr Xinyu Jiang, Department of General Surgery, Xiamen Maternity and Child Health Care Hospital, 10 Zhenhai Road, Xiamen, Fujian 361000, P.R. China

E-mail: jiangxy0818@163.com

Key words: microRNA-3934-5p, tumor protein 53-induced nuclear protein 1 , neuroblastoma, proliferation, apoptosis from the sympatoadrenal lineage, which resides in the neural crest (2-4). Unique features of neuroblastoma include the early age of onset, the high frequency of metastatic disease at diagnosis and the tendency for spontaneous regression of tumours in infancy (5). Currently, there are many methods for treating neuroblastoma including chemotherapy, radiation and autologous transplantation (6), but these treatments are unsatisfactory. The five-year survival rate of neuroblastoma in children aged 1-14 years is 68\% (7). Given such a high mortality rate, it is necessary to find an effective treatment for neuroblastoma.

MicroRNAs (miRNAs or miRs) are small non-coding RNA molecules that are generally 19-26 nucleotides in length, which serve to regulate the expression of genes at the posttranscriptional level (8-10). miRNAs do not encode protein and function to inhibit the expression of multiple target genes by binding to the target mRNA's 3' untranslated region (3'-UTR) (11-13). Previous studies have indicated that miRNAs, which are encoded by various plants, animals and viruses, can regulate many diverse biological and physiological processes including organ development, apoptosis, tumorogenesis, proliferation, stress response and fat metabolism $(14,15)$. A previous study has revealed that miR-3934 is highly expressed in cervical cancer (16). It has also been reported that miR-3934 is associated with lung adenocarcinoma (17). However, the role of miR-3934-5p in neuroblastoma remains unclear.

Tumor protein 53-induced nuclear protein 1 (TP53INP1) is a potential target gene for many miRNAs, including miR-221 (18), miR-30a (19) and miR-205 (20). Additionally, it has been reported that TP53INP1 is a regulator of autophagy and may interact with autophagy-associated molecules, including light chain 3 and autophagy related protein 8-family proteins, which indicates that it may not only regulate, but also promote autophagy $(21,22)$. Researchers have also suggested that TP53INP1 may serve an important role in certain types of cancer, including hepatocellular carcinoma (23), breast cancer (24) and human osteosarcoma (25).

In the present study, the expression of miR-3934-5p in neuroblastoma tissues and cell lines was investigated and 
the underlying mechanisms of miR-3934-5p in neuroblastoma were assessed in more detail. The association between miR-3934-5p and TP53INP1 was also investigated. The results of the present study may provide promising targets for the development of novel approaches in the management of neuroblastoma.

\section{Materials and methods}

Clinical samples. A total of 30 (Female, 12; Male, 18; age range, 3 months to 14 years) neuroblastoma tissues and normal matched adjacent tissues ( $>2 \mathrm{~cm}$ from the tumor site) were obtained from Jianou Municipal Hospital (Jianou, China) between June 2015 and December 2017. Individuals with severe concomitant diseases, including cardiovascular disease, malabsorption syndrome, liver or kidney disease, another tumor and immunodepression were excluded from the study. No patients had received any radiotherapy or chemotherapy prior to surgery. The present study was approved by the Ethical Committee of Jianou Municipal Hospital (Jianou, China). Written informed consent was obtained from each patient and their parent or guardians.

Cell culture and cell transfection. Human neuroblastoma cell lines CHLA-20, CHLA-15 and SMS-KAN (Children's Oncology Group, Cell Culture and Xenograft Repository, Texas Tech University Health Science Centre; Lubbock, TX, USA) were cultured in Iscove's Modified Dulbecco's Medium (Sigma-Aldrich; Merck KGaA, Darmstadt, Germany). SK-N-SH (cat. no. ATCC ${ }^{\circledR}$ HTB-11 ${ }^{\text {TM }}$; American Type Culture Collection, Manassas, VA, USA) were cultured in Eagle's Minimum Essential medium (EMEM; Gibco; Thermo Fisher Scientific, Inc.) supplemented with 10\% FBS (Gibco; Thermo Fisher Scientific, Inc.) at $37^{\circ} \mathrm{C}$ for $24 \mathrm{~h}$. Human umbilical vein endothelial cells (HUVECs) were also cultured in M199 medium (Gibco; Thermo Fisher Scientific, Inc.) supplemented with 20\% FBS (Gibco; Thermo Fisher Scientific, Inc.).

On the day prior to cell transfection, SK-N-SH cells were plated into a six-well plate at a density of $1 \times 10^{6}$ cells per well and cultured at $37^{\circ} \mathrm{C}$ with $5 \% \mathrm{CO}_{2}$. SK-N-SH cells were transfected with $50 \mathrm{nM}$ inhibitor control [cat. no. CS8005; Biomics Biotechnologies (Nantong) Co., Ltd.], 50 nM miR-3934-5p inhibitor [cat. no. hsa-miR-3934-5p; Biomics Biotechnologies (Nantong) Co., Ltd], $2 \mu \mathrm{l}$ control-small interfering RNA (siRNA; cat. no. sc-36869; Santa Cruz Biotechnology, Inc.), $2 \mu 1$ TP53INP1-siRNA (cat. no. sc-76715; Santa Cruz Biotechnology, Inc.) or $50 \mathrm{nM}$ miR-3934-5p inhibitor+2 $\mu 1$ TP53INP1-siRNA using Lipofectamine 2000 reagent (Invitrogen; Thermo Fisher Scientific, Inc.) according to the manufacturer's protocol. $48 \mathrm{~h}$ later, the transfection efficiency was detected using reverse transcription-quantitative (RT-q) PCR.

$R T-q P C R$. Total RNA from tissues and cells was extracted using the TRIzol reagent (Invitrogen; Thermo Fisher Scientific, Inc.) according to the manufacturer's protocol. Total RNA concentration was then detected via Nanodrop2000 (Thermo Fisher Scientific, Inc.) and stored at $-80^{\circ} \mathrm{C}$ until further use. The synthesis of cDNA was performed using the RevertAid ${ }^{\mathrm{TM}}$ First Strand cDNA Synthesis kit (Thermo Fisher Scientific, Inc.) according to the manufacturer's protocol. qPCR was subsequently performed using the SYBR Premix Ex TaqTM II (TliRNaseH Plus) kit (Takara Bio, Inc.). The amplification conditions for qPCR were as follows: $10 \mathrm{~min}$ at $95^{\circ} \mathrm{C}$ followed by 35 cycles of $15 \mathrm{sec}$ at $95^{\circ} \mathrm{C}, 40 \mathrm{sec}$ at $55^{\circ} \mathrm{C}$ and $72^{\circ} \mathrm{C}$ for $30 \mathrm{sec}$. The primer sequences used for $\mathrm{qPCR}$ were as follows: TP53INP1 forward, 5'-GACTCACGGGCACAGAAGTGG AAGC-3' and reverse, 5'-CCACTGGGAAGGGCGAAAG-3'; GAPDH forward, 5'-CTTTGGTATCGTGGAAGGACTC-3' and reverse, 5'-GTAGAGGCAGGGATGATGTTCT-3'; U6 forward, 5'-CGCTTCACGAATTTGCGTGTCAT-3'; Bcl-2 forward, 5'-TTGGATCAGGGAGTTGGAAG-3' and reverse, 5'-TGTCCCTACCAACCAGAAGG-3'; Bax forward, 5'-CGT CCACCAAGAAGCTGAGCG-3' and reverse, 5'-CGTCCA CCAAAGCTGAGCG3-3'; Cyclin dependent kinase inhibitor 1A (p21) forward, 5'-TGAGCCGCGACTGTGATG-3' and reverse, 5'-GTCTCGGTGACAAAGTCGAAGTT-3'. The relative expression of genes were calculated using the $2^{-\Delta \Delta C q}$ method (26) following normalization with reference to the expression of GAPDH or U6. All experiments were performed in triplicate to ensure for minimum deviation.

Western blotting. Cells were washed three times with cold PBS and total cellular proteins were extracted using Radioimmunoprecipitation assay (RIPA) lysis buffer (Beyotime Institute of Biotechnology) at $4^{\circ} \mathrm{C}$ for $30 \mathrm{~min}$. The bicinchoninic acid protein assay kit (Beyotime Institute of Biotechnology) was used for protein quantification. Equal quantities of protein (30 $\mu \mathrm{g}$ per lane) were separated on $10 \%$ SDS-PAGE gel and transferred to PVDF membranes. The membranes were then blocked with $5 \%$ non-fat milk at room temperature for $2 \mathrm{~h}$. This was then followed by incubation with primary antibodies against TP53INP1 (1:1,000; cat. no. A04229; Wuhan Boster Biological Technology, Ltd.), Bcl-2 (1:1,000; cat. no. 4223; Cell Signaling Technology, Inc.), Bax (1:1,000; cat. no. 5023; Cell Signaling Technology, Inc.), p21 (1:1,000; cat. no. 2947; Cell Signaling Technology, Inc.) or $\beta$-actin (1:1,000; cat. no. 4970; Cell Signaling Technology, Inc.) overnight at $4^{\circ} \mathrm{C}$. The membranes were then incubated with horseradish peroxidase-conjugated secondary anti-rabbit IgG antibody (1:2,000; cat. no. 7074; Cell Signaling Technology, Inc.), for $2 \mathrm{~h}$ at room temperature. Finally, protein bands were visualized using the Western Blotting Luminol Reagent (cat. no. sc-2048; Santa Cruz Biotechnology, Inc.) according to the manufacturer's protocol.

Cell counting kit-8 (CCK-8) assay. A CCK-8 assay was performed to measure cell viability. Logarithmic phase cells were seeded in a 96-well plate with $1 \times 10^{4}$ cells per well and incubated in $37^{\circ} \mathrm{C}$ with $5 \% \mathrm{CO}_{2}$ for $12 \mathrm{~h}$, after which $10 \mu \mathrm{l}$ CCK-8 solution (Beyotime Institute of Biotechnology) was added to each well and cells were incubated for a further $2 \mathrm{~h}$ at $37^{\circ} \mathrm{C}$ with $5 \% \mathrm{CO}_{2}$. Absorbance was measured at a wavelength of $450 \mathrm{~nm}$ using a FLUOstar ${ }^{\circledR}$ Omega Microplate Reader to assess cell viability (BMG Labtech $\mathrm{GmbH})$.

Flow cytometry assay. Cells were collected in the logarithmic growth phase via trypsinization, washed three times with PBS and then trypsinized into single cell suspensions. Apoptotic cells were detected using the Annexin V-(FITC)/propidium iodide (PI) apoptosis detection kit [cat. no. 70-AP101-100; 


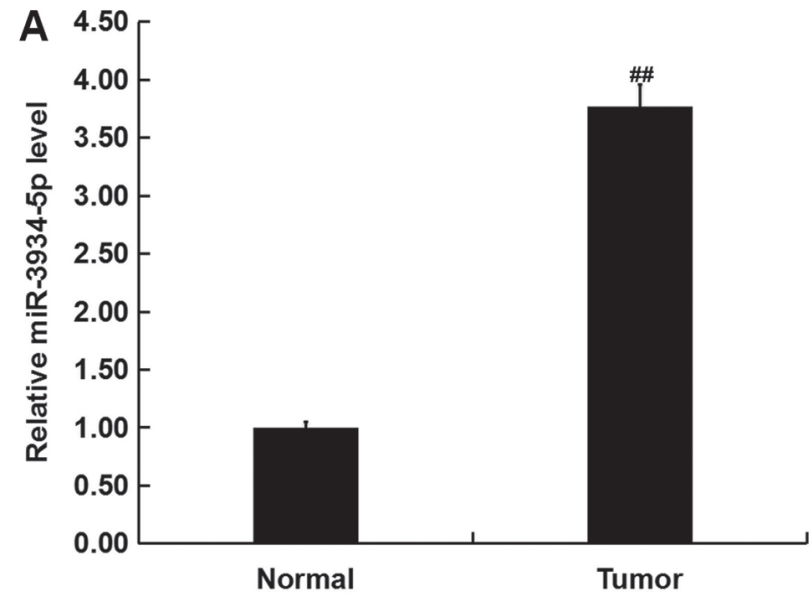

B

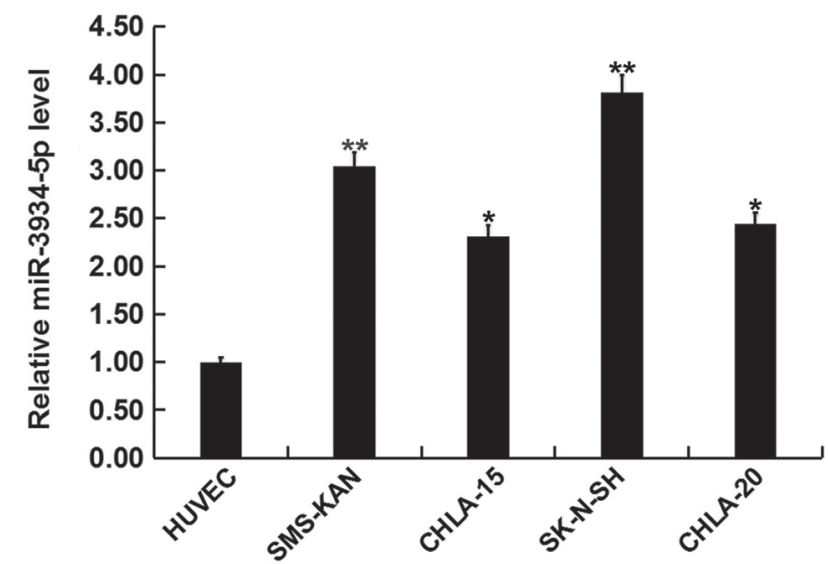

Figure 1. miR-3934-5p was upregulated in neuroblastoma tissues and cell lines. (A) The relative expression of miR-3934-5p in neuroblastoma tissues and normal matched adjacent tissue was determined via RT-qPCR. (B) The relative expression of miR-3934-5p in neuroblastoma cell lines, including CHLA-20, CHLA-15, SMS-KAN, SK-N-SH and non-malignant neuroblastoma cell HUVECs was determined via RT-qPCR. All data are presented as the mean \pm standard deviation of three independent experiments. "P<0.05 and ${ }^{* *} \mathrm{P}<0.01$ vs. HUVEC; ${ }^{\# \#} \mathrm{P}<0.01$ vs. Normal. miR-3934-5p, microRNA-3934-5p; RT-qPCR, reverse transcription-quantitative PCR; HUVEC, human umbilical vein endothelial cells; Tumor, neuroblastoma tissue; Normal, normal matched adjacent tissue.

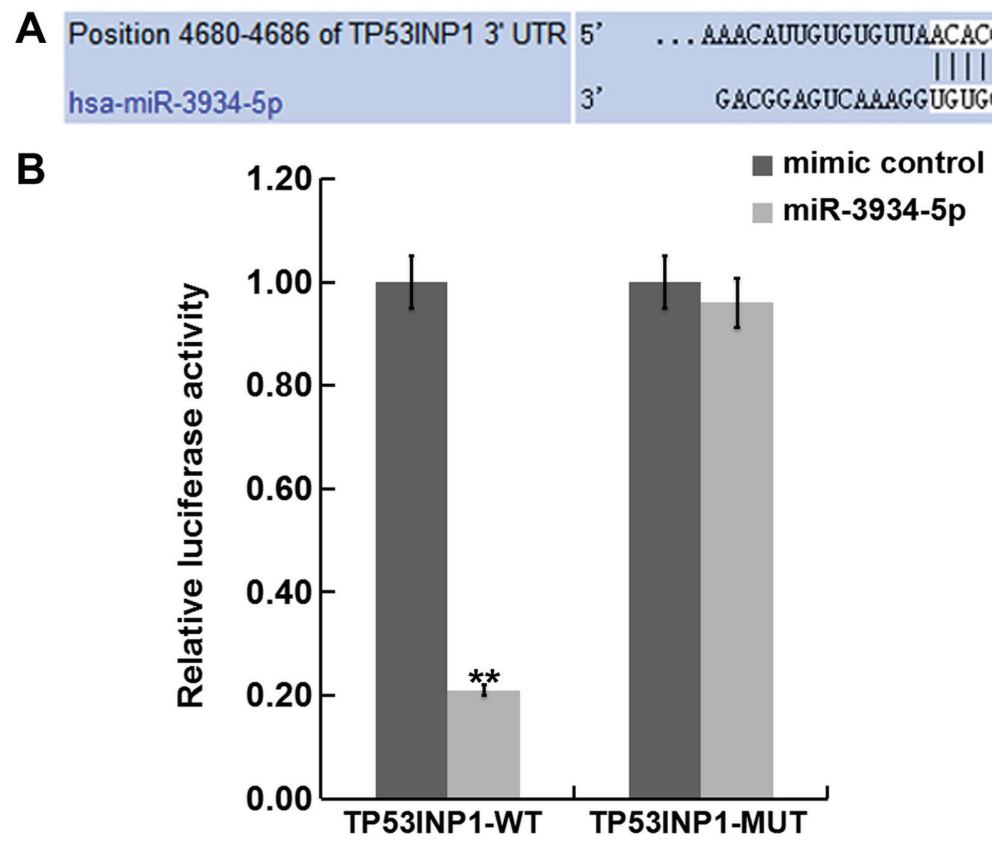

Figure 2. TP53INP1 was the direct gene of miR-3934-5p. (A) TargetScan predicted TP53INP1 the 3'-UTR binding sites for miR-3934-5p. (B) Luciferase activity of a reporter containing a TP53INP1-WT 3'UTR or a TP53INP1-MUT 3'UTR are presented. All data are presented as the mean \pm standard deviation of three independent experiments. ${ }^{* *} \mathrm{P}<0.01$ vs. mimic control. TP53INP1, tumor protein 53-induced nuclear protein 1; miR-3934-5p, microRNA-3934-5p; UTR, untranslated region; WT, wild-type; MUT, mutant.

Hangzhou MultiSciences (Lianke) Biotech Co.,Ltd.] according to the manufacturer's instructions. Cells were stained with $5 \mu \mathrm{l}$ Annexin V-FITC and $5 \mu \mathrm{l}$ PI for $30 \mathrm{~min}$ for $15 \mathrm{~min}$ in darkness at room temperature. Flow cytometry was performed (BD Biosciences) according to the manufacturer's protocol to detect cell apoptosis. The apoptotic rate was determined using FlowJo software version 7.6.1 (FlowJo LLC).

Dual-luciferase reporter assay. To predict the targets of miR-3934-5p, TargetScan bioinformatics software (www. targetscan.org/vert_71) was applied. To investigate the association between miR-3934-5p and TP53INP1, the wild type (WT-TP53INP1) and mutant (MUT-TP53INP1) 3'-UTRs of TP53INP1 were cloned into a pmiR-RB-ReportTM dual luciferase reporter gene plasmid vector (Guangzhou RiboBio Co., Ltd.) as per the manufacturer's instructions. Cells seeded in 24 -well plates $\left(5 \times 10^{4}\right.$ cells per well) were then co-transfected with miR-3934-5p mimics or mimic controls and the MUT or WT of TP53INP1 using the Lipofectamine 2000 reagent (Invitrogen; Thermo Fisher Scientific, Inc.) for 48 h, together 
A
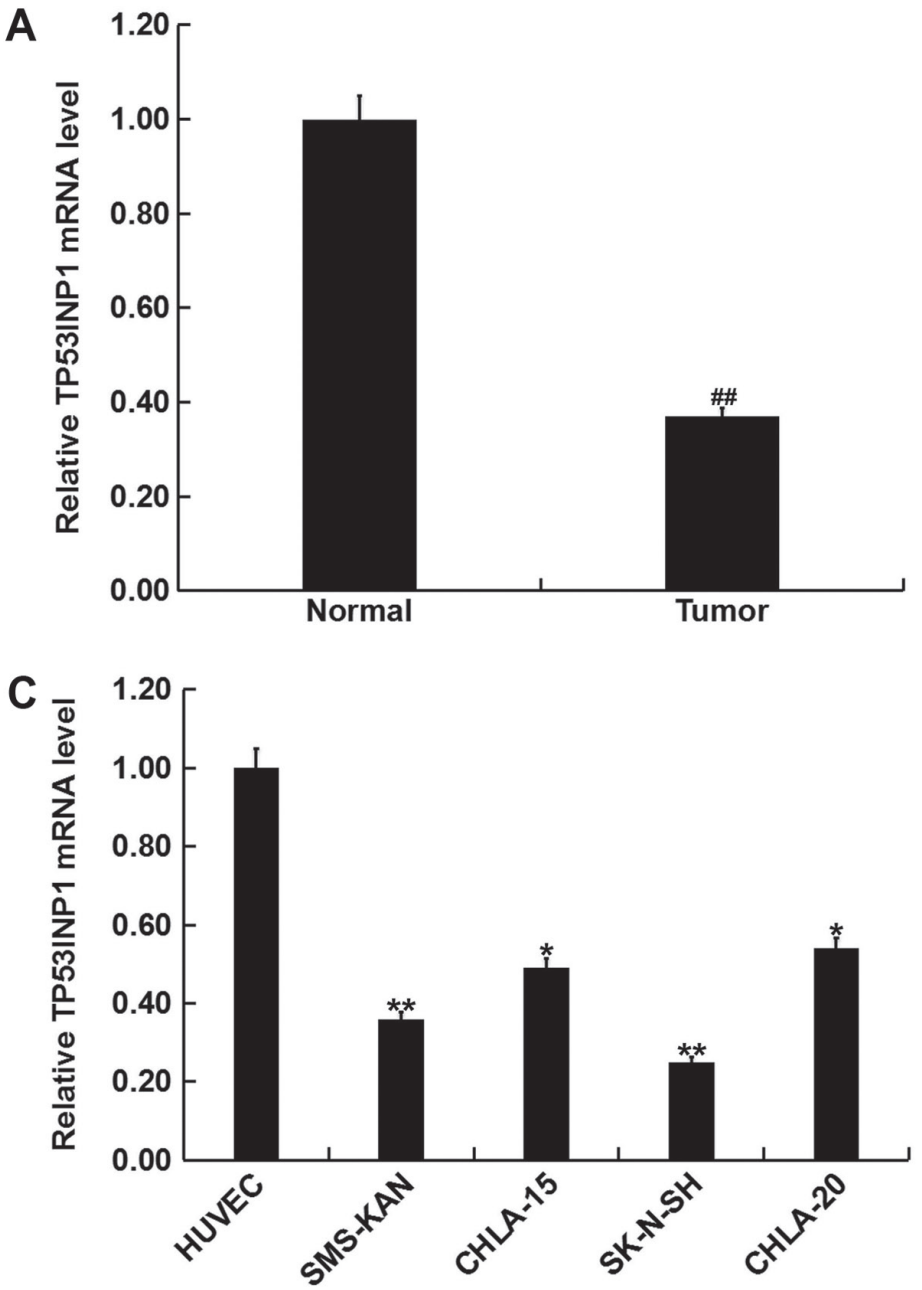

B

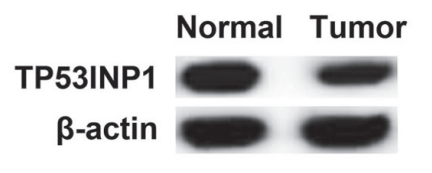

D

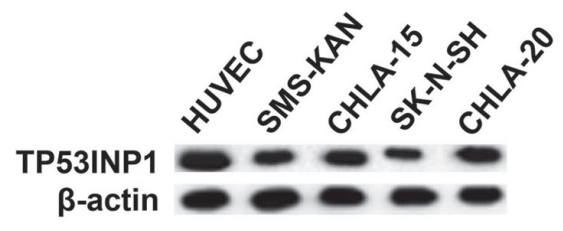

Figure 3. TP53INP1 was downregulated in neuroblastoma tissues and cell lines. (A) RT-qPCR and (B) western blot analysis were performed to detect the relative mRNA and protein expression of TP53INP1 in neuroblastoma tissues and normal matched adjacent tissue. (C) RT-qPCR (D) and western blot analysis were performed to detect the relative mRNA and protein expression of TP53INP1 in neuroblastoma cells lines (CHLA-20, CHLA-15, SMS-KAN and SK-N-SH) and HUVEC. All data were presented as the mean \pm standard deviation of three independent experiments. "P<0.05, ${ }^{* *} \mathrm{P}<0.01$ vs. HUVEC; ${ }^{\# \#} \mathrm{P}<0.01$ vs. Normal. TP53INP1, tumor protein 53-induced nuclear protein 1; RT-qPCR, reverse transcription-quantitative PCR; HUVEC, human umbilical vein endothelial cells; Tumor, neuroblastoma tissue; Normal, normal matched adjacent tissue.

with the Renilla luciferase pRL-TK vector as a control. After transfection for $48 \mathrm{~h}$, cells were lysed with RIPA buffer (Beyotime Institute of Biotechnology). Relative luciferase activity was detected using the dual-luciferase reporter assay system (Promega Corporation) according to the manufacturer's instructions. Luciferase activity was normalized to that of Renilla luciferase activity.

Statistical analysis. Each experiment was performed at least three times. All data was presented as the mean \pm standard deviation. Significant differences multiple groups were measured using one-way ANOVA with a Tukey's post hoc test, and the Student's t-test was used to perform comparisons between two groups. $\mathrm{P}<0.05$ was considered to indicate a statistically significant difference. Data analyses were performed using SPSS software version 17.0 (SPSS, Inc.).

\section{Results}

Expression of miR-3934-5p in neuroblastoma tissues and cell lines. To assess the role of miR-3934-5p in neuroblastoma, the level of miR-3934-5p in neuroblastoma and normal matched adjacent tissues were detected using RT-qPCR. The results indicated that the expression of miR-3934-5p was upregulated in neuroblastoma tissues compared with normal matched adjacent tissues (Fig. 1A). miR-3934-5p levels in different neuroblastoma cell lines (CHLA-20, CHLA-15, SMS-KAN and SK-N-SH) and HUVECs were also measured. The current study demonstrated that the expression of miR-3934-5p was significantly upregulated in CHLA-20, CHLA-15, SMS-KAN and SK-N-SH cells in comparison with HUVEC. The highest level of miR-3934-5p was observed in SK-N-SH cells (Fig. 1B).

TP53INP1 is a direct gene of miR-3934-5p. In order to determine the interaction between miRNAs and their target genes, TargetScan was used to predict the target gene of miR-3934-5p. The software revealed that the TP53INP1 3'UTR of mRNA contains a putative site that is partially complementary to miR-3934-5p (Fig. 2A). A luciferase reporter assay was performed to examine if miR-3934-5p interacts directly with the target gene TP53INP1. Compared with co-transfection of MUT-TP53INP1 and miR-3934-5p mimics, luciferase activity 
A

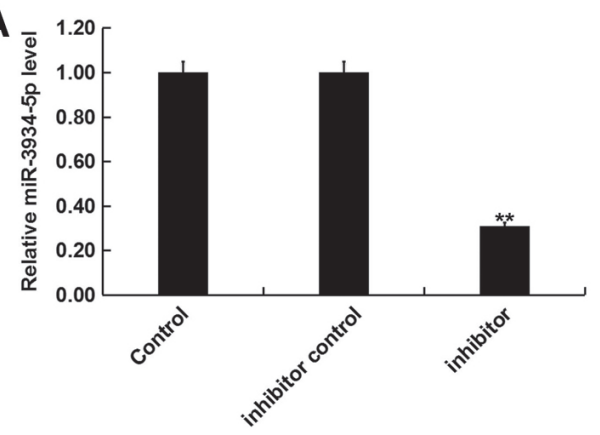

D

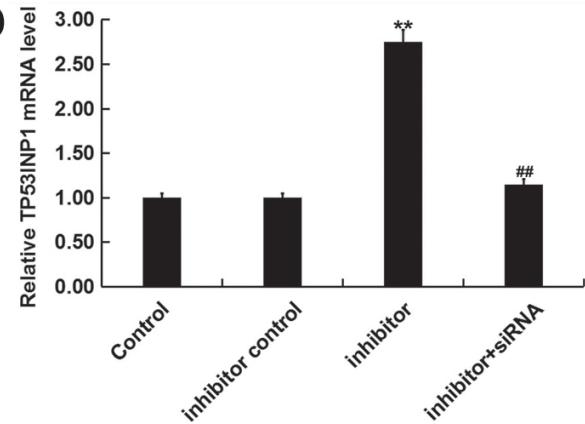

B

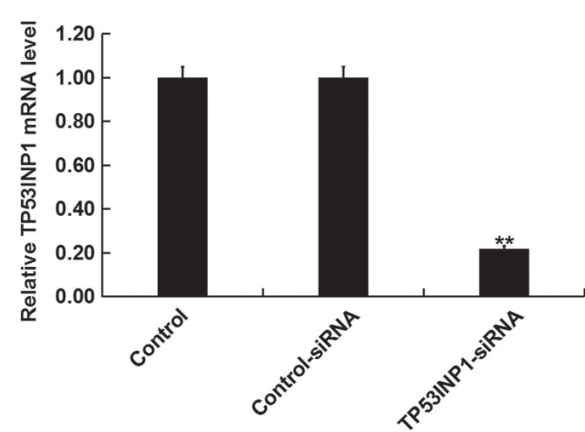

E
C

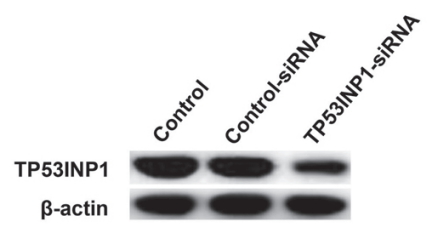

Figure 4. miR-3934-5p negatively regulated the expression of TP53INP1 in SK-N-SH cells. (A) RT-qPCR was performed to measure the expression of miR-3934-5p in SK-N-SH cells transfected with an inhibitor control or a miR-3934-5p inhibitor for 48 h. (B) RT-qPCR and (C) a western blot assay were performed to measure the mRNA and protein expression of TP53INP1 in SK-N-SH cells transfected with control-siRNA or TP53INP1-siRNA for 48 h. (D) RT-qPCR and (E) a western blot analysis were performed to measure the mRNA and protein expression of TP53INP1 in SK-N-SH cells transfected with an inhibitor control, a miR-3934-5p inhibitor, or a miR-3934-5p inhibitor+TP53INP1-siRNA for $48 \mathrm{~h}$. All data are presented as the mean \pm standard deviations of three independent experiments. ${ }^{* *} \mathrm{P}<0.01$ vs. Control; ${ }^{\# \#} \mathrm{P}<0.01$ vs. inhibitor. miR-3934-5p, microRNA-3934-5p; TP53INP1, Tumor protein 53-induced nuclear protein 1; RT-qPCR, reverse transcription-quantitative PCR; siRNA, small interfering RNA.
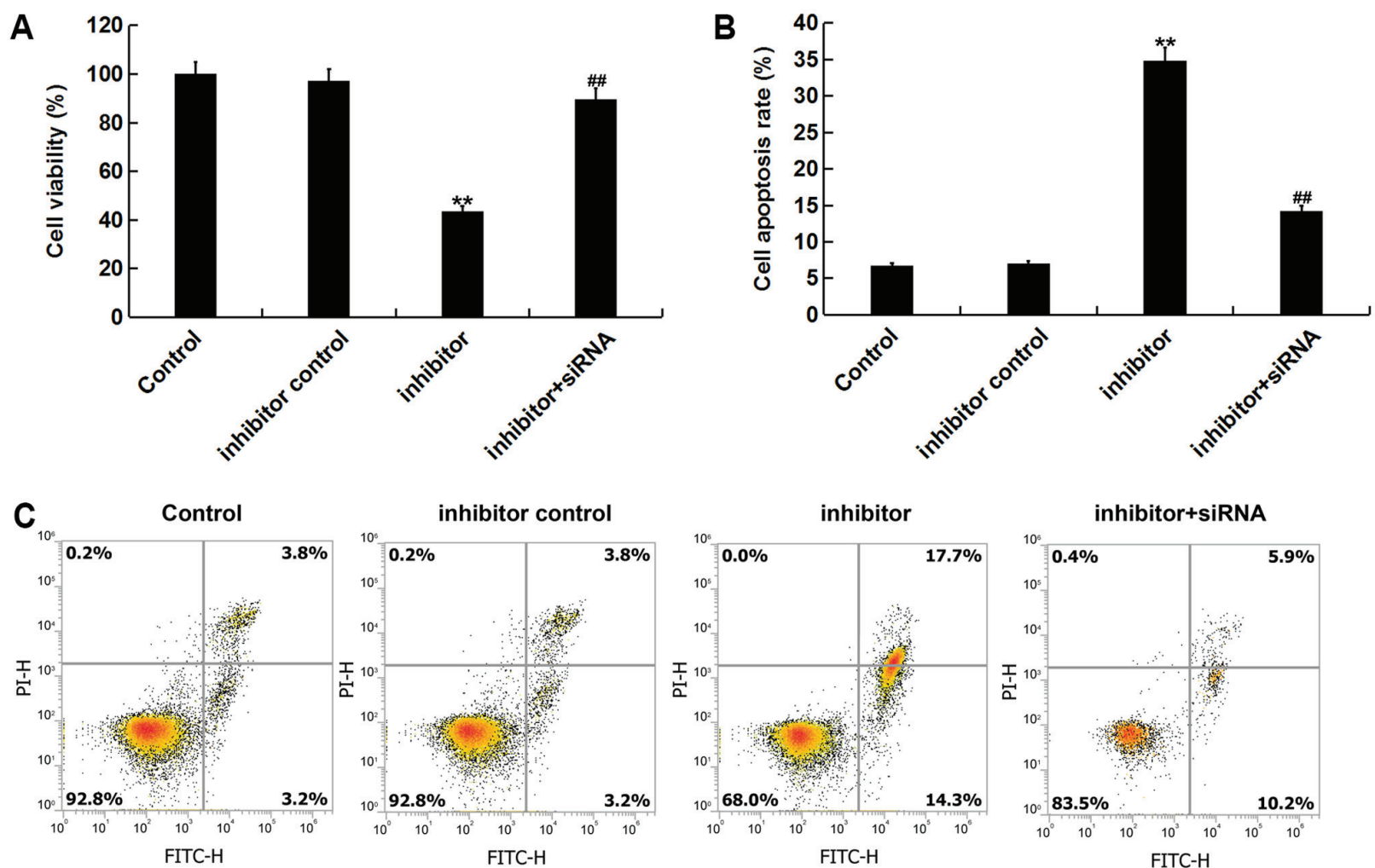

Figure 5. Effect of miR-3934-5p on cell viability and apoptosis in SK-N-SH cells. (A) A cell counting kit-8 assay was used to detect cell viability after SK-N-SH cells were transfected with an inhibitor control, a miR-3934-5p inhibitor, or a miR-3934-5p inhibitor+TP53INP1-siRNA for 48 h. (B) Cell apoptosis rate was determined via (C) flow cytometry after SK-N-SH cells were transfected with an inhibitor control, a miR-3934-5p inhibitor, or a miR-3934-5p inhibitor+TP53INP1-siRNA for $48 \mathrm{~h}$. All data are presented as the mean \pm standard deviation of three independent experiments. ${ }^{* *} \mathrm{P}<0.01$ vs. Control; ${ }^{\# \prime} \mathrm{P}<0.01$ vs. inhibitor. miR-3934-5p, microRNA-3934-5p; CCK-8, cell counting kit-8; TP53INP1, tumor protein 53-induced nuclear protein 1; siRNA, small interfering RNA. 
A

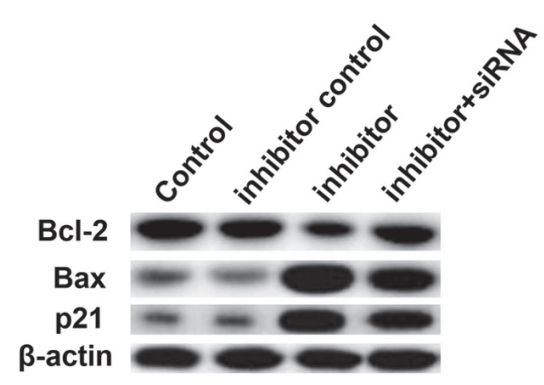

B

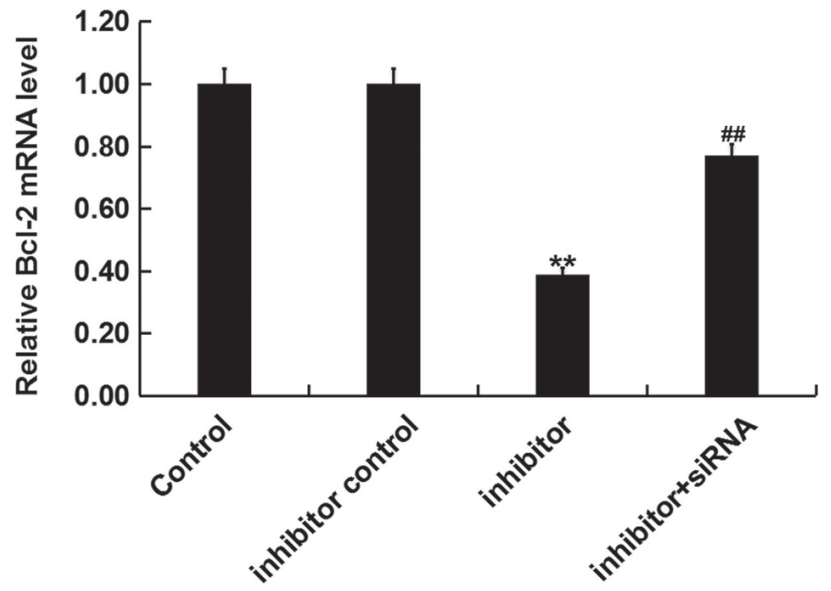

C

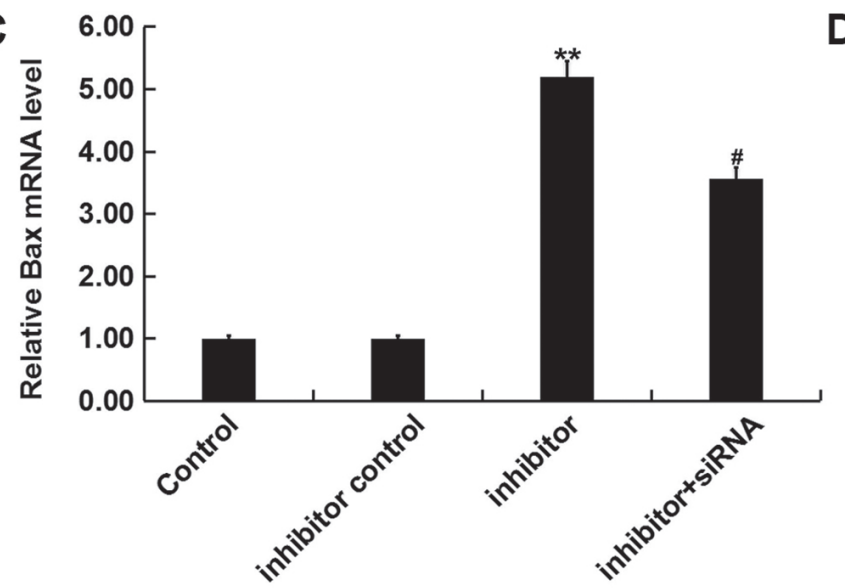

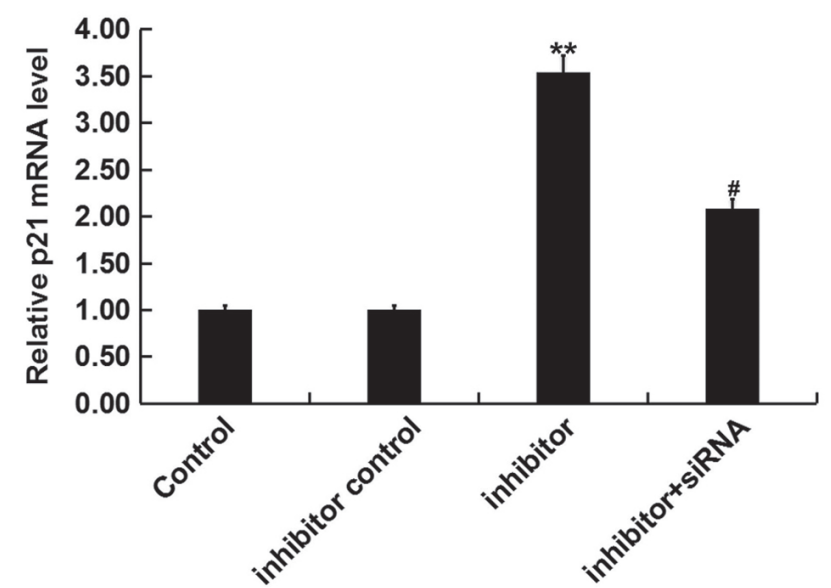

Figure 6. Effect of miR-3934-5p on the expression of Bcl-2, Bax and p21 in SK-N-SH cells. SK-N-SH cells were transfected with an inhibitor control, a miR-3934-5p inhibitor, or an miR-3934-5p inhibitor+TP53INP1-siRNA for $48 \mathrm{~h}$. (A) Western blot analysis and reverse transcription-quantitative PCR were performed to measure the protein and mRNA expression of (B) Bcl-2, (C) Bax and (D) p21. All data are presented as the mean \pm standard deviation of three independent experiments. ${ }^{* *} \mathrm{P}<0.01$ vs. Control; ${ }^{\#} \mathrm{P}<0.05$ and ${ }^{\# \#} \mathrm{P}<0.01$ vs. inhibitor. miR-3934-5p, microRNA-3934-5p; p21, cyclin dependent kinase inhibitor $1 \mathrm{~A}$.

was significantly decreased following co-transfection with WT-TP53INP1 and miR-3934-5p mimics (Fig. 2B). These results suggest that TP53INP1 may be a direct target gene of miR-3934-5p.

Expression of TP53INP1 in neuroblastoma tissues and cell lines. The differences in expression of TP53INP1 between neuroblastoma tissues and normal matched adjacent tissues were determined using RT-qPCR and western blotting. The data revealed that TP53INP1 was downregulated in neuroblastoma tissues compared with normal matched adjacent tissues (Fig. 3A and B). In addition, RT-qPCR and western blotting revealed that compared with HUVEC, TP53INP1 was significantly downregulated in neuroblastoma cell lines, including CHLA-20, CHLA-15, SMS-KAN and SK-N-SH at both mRNA and protein levels. The lowest expression was observed in SK-N-SH cells (Fig. 3C and D). The results indicate that there is an inverse association between the expression of miR-3934-5p and TP53INP1 in neuroblastoma.

miR-3934-5p negatively regulates the expression of TP53INP1 in SK-N-SH cells. SK-N-SH cells were transfected with inhibitor controls, miR-3934-5p inhibitors,
control-siRNA, TP53INP1-siRNA, or miR-3934-5p inhibitor+TP53INP1-siRNA for $48 \mathrm{~h}$. RT-qPCR or western blot analysis was then performed to detect transfection efficiency. The RT-qPCR assay revealed that the miR-3934-5p inhibitor significantly reduced the expression of miR-3934-5p in SK-N-SH cells (Fig. 4A). RT-qPCR and western blot analysis also indicated that TP53INP1-siRNA decreased the mRNA and protein expression of TP53INP1 in SK-N-SH cells (Fig. 4B and C). The results also indicated that the miR-3934-5p inhibitor significantly increased the mRNA and protein expression of TP53INP1. However, this effect was reversed via TP53INP1-siRNA treatment (Fig. 4D and E).

Effect of miR-3934-5p on viability and apoptosis of neuroblastoma cells. To assess the role of miR-3934-5p in neuroblastoma cells, the effect of miR-3934-5p on the viability of SK-N-SH cells was assessed. The result of the CCK-8 assay indicated that compared with the control group, the miR-3934-5p inhibitor significantly decreased cell viability. This decrease was subsequently reversed by TP53INP1-siRNA treatment (Fig. 5A). To determine the apoptotic effect of miR-3934-5p, flow cytometry was performed. Analysis revealed that transfection with the miR-3934-5p 
inhibitor significantly induced cell apoptosis. This apoptotic effect was then reversed via TP53INP1-siRNA treatment (Fig. 5B and C). Furthermore, RT-qPCR and western blot analysis revealed that the miR-3934-5p inhibitor significantly decreased Bcl-2 protein and mRNA expression, and increased Bax and $\mathrm{p} 21$ expression, which was then reversed via TP53INP1-siRNA (Fig. 6A and D).

\section{Discussion}

It has been revealed that miR-3934 may be associated with cervical and lung cancer $(16,17)$. The current study demonstrated that miR-3934-5p is highly expressed in both neuroblastoma tissues and cell lines compared with adjacent normal tissues and cells. miRNAs serve important roles in many cell development processes, including cell proliferation, cell differentiation and apoptosis (27-32). Increasing evidence has also revealed that the aberrant expression of miRNAs may be associated with different types of cancer, in which miRNAs may act as tumor suppressors or oncogenes (33-37).

TP53INP1 is a p53-inducible gene that encodes for two protein isoforms, which modulate p53 biological activities (38). TP53INP1 over-expression induces cell cycle arrest in the G1 phase and enhances p53-mediated apoptosis (39). Many studies have demonstrated that TP53INP1 is a target gene of many miRNAs (18-20,25,40-42). To the best of our knowledge, there are no studies regarding the association between TP53INP1 and miR-3934-5p in neuroblastoma. In certain types of cancer, TP53INP1 functions as a tumor suppressor $(21,43)$. Within the current study, the dual luciferase reporter assay indicated that miR-3934-5p directly targets TP53INP1 and that TP53INP1 was downregulated in neuroblastoma tissues and cell lines. These results indicated that there was an inverse association between the expression of miR-3934-5p and TP53INP1 in neuroblastoma. However, in prostate cancer, TP53INP1 has been reported to act as an oncogene and its over-expression is associated with castration-resistant prostate cancer (44). Several miRNAs have been indicated to negatively regulate TP53INP1 expression including, miR-155 in pancreatic cancer (45), miR-130b in hepatocellular cancer (41), miR-125b in type 2 endometrial carcinoma cells (42), and miR-569 in epithelial cancers (46). In the present study, HUVECs were used as control cells. However, whether the expression of miR-3934-5p and TP53INP1 in endothelial cells can be representative of other normal cells requires further study.

To investigate the effects of miR-3934-5p and TP53INP1 on neuroblastoma cell proliferation and apoptosis, SK-N-SH cells were transfected with an inhibitor control, a miR-3934-5p inhibitor, or miR-3934-5p inhibitor+TP53INP1-siRNA for $48 \mathrm{~h}$. The results of the CCK-8 assay and flow cytometry revealed that the miR-3934-5p inhibitor inhibited cell viability and induced cell apoptosis. In addition, the expression of the anti-apoptotic gene Bcl-2, the pro-apoptotic gene Bax and the apoptosis regulator p21 were also determined in the current study. The findings of the current study indicate that the miR-3934-5p inhibitor significantly decreased Bcl-2 increased Bax and increased p21 expression at both protein and mRNA levels. Furthermore, all the effects of the miR-3934-5p inhibitor on neuroblastoma cells were reversed via TP53INP1-siRNA treatment.
In summary, the data of the current study demonstrated that miR-3934-5p was downregulated in neuroblastoma tissues and cell lines. miR-3934-5p downregulation also significantly inhibited neuroblastoma cell viability and induced apoptosis by directly regulating the expression of TP53INP1. The present study has therefore provided a potential novel therapeutic target for neuroblastoma.

\section{Acknowledgements}

The authors would like to acknowledge Dr Yang Jie and Dr Huang Rongbin (Science and Education Department, Jianou Municipal Hospital, Jianou, China) who offered valuable suggestions for the present study.

\section{Funding}

No funding was received.

\section{Availability of data and materials}

The datasets used and/or analyzed during the current study are available from the corresponding author on reasonable request.

\section{Authors' contributions}

WY contributed to the study design, data collection, statistical analysis, data interpretation and manuscript preparation. FL, CY and MZ contributed to data collection and data interpretation. DF and XJ contributed to the statistical analysis and performed the literature search.

\section{Ethics approval and consent to participate}

The present study was approved by the Ethical Committee of Jianou Municipal Hospital (Jianou, China). Written informed consent was obtained from each patient and their parent or guardians.

\section{Patient consent for publication}

All patients provided consent to publish data.

\section{Competing interests}

The authors declare that they have no competing interests.

\section{References}

1. Health Professional Version: Neuroblastoma Treatment (PDQ $\left.{ }^{\circledR}\right)-H e a l t h$ Professional Version. National Cancer Institute, Bethesda, MD, 2014. https://www.cancer.gov/types/neuroblastoma/hp/neuroblastoma-treatment-pdq.

2. Gatta G, Botta L, Rossi S, Aareleid T, Bielska-Lasota M, Clavel J, Dimitrova N, Jakab Z, Kaatsch P, Lacour B, et al: Childhood cancer survival in Europe 1999-2007: Results of EUROCARE-5-a population-based study. Lancet Oncol 15: 35-47, 2014.

3. Brodeur GM and Bagatell R: Mechanisms of neuroblastoma regression. Nat Rev Clin Oncol 11: 704-713, 2014.

4. Li P, Gao Y, Ji Z, Zhang X, Xu Q, Li G, Guo Z, Zheng B and Guo X: Role of urokinase plasminogen activator and its receptor in metastasis and invasion of neuroblastoma. J Pediatr Surg 39: 1512-1519, 2004. 
5. Matthay KK, Maris JM, Schleiermacher G, Nakagawara A, Mackall CL, Diller L and Weiss WA: Neuroblastoma. Nat Rev Dis Primers 2: 16078, 2016.

6. Mazar J, Li Y, Rosado A, Phelan P, Kedarinath K, Parks GD, Alexander KA and Westmoreland TJ: Zika virus as an oncolytic treatment of human neuroblastoma cells requires CD24. PLoS One 13: e0200358, 2018.

7. Smith MA, Altekruse SF, Adamson PC, Reaman GH and Seibel NL: Declining childhood and adolescent cancer mortality. Cancer 120: 2497-2506, 2014.

8. Ambros V: The functions of animal microRNAs. Nature 431: 350-355, 2004

9. Bartel DP: MicroRNAs: Genomics, biogenesis, mechanism, and function. Cell 116: 281-297, 2004.

10. Kwon C, Han Z, Olson EN and Srivastava D: MicroRNA1 influences cardiac differentiation in Drosophila and regulates Notch signaling. Proc Natl Acad Sci USA 102: 18986-18991, 2005.

11. Ro S,Park C, Young D, Sanders KM and Yan W: Tissue-dependent paired expression of miRNAs. Nucleic Acids Res 35: 5944-5953, 2007.

12. Mallory AC and Vaucheret H: MicroRNAs: Something important between the genes. Curr Opin Plant Biol 7: 120-125, 2004.

13. Garzon R, Calin GA and Croce CM: MicroRNAs in cancer. Annu Rev Med 60: 167-179, 2009.

14. Ambros V: microRNAs: Tiny regulators with great potential. Cell 107: 823-826, 2001.

15. Carrington JC and Ambros V: Role of microRNAs in plant and animal development. Science 301: 336-338, 2001.

16. Chen JY, Yao DS, He CJ, et al: Differential expression of microRNA in cervical squamous cell carcinoma. J Pract Med 30: 83-87, 2014.

17. Sathipati SY and Ho SY: Identifying the miRNA signature associated with survival time in patients with lung adenocarcinoma using miRNA expression profiles. Sci Rep 7, 2017.

18. Chen Q, Zhou Y, Richards AM and Wang P: Up-regulation of miRNA-221 inhibits hypoxia/reoxygenation-induced autophagy through the DDIT4/mTORC1 and Tp53inp1/p62 pathways. Biochem Biophys Res Commun 474: 168-174, 2016.

19. Xu CG, Yang MF, Fan JX and Wang W: MiR-30a and miR-205 are downregulated in hypoxia and modulate radiosensitivity of prostate cancer cells by inhibiting autophagy via TP53INP1. Eur Rev Med Pharmacol Sci 20: 1501-1508, 2016.

20. Wang W, Liu J and Wu Q: MiR-205 suppresses autophagy and enhances radiosensitivity of prostate cancer cells by targeting TP53INP1. Eur Rev Med Pharmacol Sci 20: 92-100, 2016.

21. Seillier M, Peuget S, Gayet O, Gauthier C, N'Guessan P, Monte M, Carrier A, Iovanna JL and Dusetti NJ: TP53INP1, a tumor suppressor, interacts with LC3 and ATG8-family proteins through the LC3-interacting region (LIR) and promotes autophagy-dependent cell death. Cell Death Differ 19: 1525-1535, 2012 .

22. Saadi H, Seillier M and Carrier A: The stress protein TP53INP1 plays a tumor suppressive role by regulating metabolic homeostasis. Biochimie 118: 44-50, 2015.

23. Xue X, Wang X, Zhao Y, Hu R and Qin L: Exosomal miR-93 promotes proliferation and invasion in hepatocellular carcinoma by directly inhibiting TIMP2/TP53INP1/CDKN1A. Biochem Biophys Res Commun 502: 515-521, 2018.

24. Wang Y, Sun H, Zhang D, Fan D, Zhang Y, Dong X, Liu S, Yang Z, Ni C, Li Y, et al: TP53INP1 inhibits hypoxia-induced vasculogenic mimicry formation via the ROS/snail signalling axis in breast cancer. J Cell Mol Med 22: 3475-3488, 2018.

25. Cai Q, Zeng S, Dai X, Wu J and Ma W: miR-504 promotes tumour growth and metastasis in human osteosarcoma by targeting TP53INP1. Oncol Rep 38: 2993-3000, 2017.

26. Livak KJ and Schmittgen TD: Analysis of relative gene expression data using real-time quantitative PCR and the 2(-Delta Delta C(T)) method. Methods 25: 402-408, 2001.

27. Yu X, Li Z, Shen J, Wu WK, Liang J, Weng X and Qiu G: MicroRNA-10b promotes nucleus pulposus cell proliferation through RhoC-Akt pathway by targeting HOXD10 in intervetebral disc degeneration. PLoS One 8: e83080, 2013.

28. Li Z, Yu X, Shen J, Wu WK and Chan MT: MicroRNA expression and its clinical implications in Ewing's sarcoma. Cell Prolif 48: $1-6,2015$.
29. Yu X and Li Z: MicroRNAs regulate vascular smooth muscle cell functions in atherosclerosis (review). Int J Mol Med 34: 923-933, 2014.

30. Li M, Yu M, Liu C, Zhu H, He X, Peng S and Hua J: miR-34c works downstream of p53 leading to dairy goat male germline stem-cell (mGSCs) apoptosis. Cell Prolif 46: 223-231, 2013.

31. Wang Z, Wang N, Liu P, Chen Q, Situ H, Xie T, Zhang J, Peng C, Lin Y and Chen J: MicroRNA-25 regulates chemoresistance-associat-ed autophagy in breast cancer cells, a process modulated by the natural autophagy inducer isoliquiritigenin. Oncotarget 5: 7013-7026, 2014.

32. Dong R, Liu X, Zhang Q, Jiang Z, Li Y, Wei Y, Li Y, Yang Q, Liu J, Wei JJ, et al: miR-145 inhibits tumor growth and metastasis by targeting metadherin in high-grade serous ovarian carcinoma. Oncotarget 5: 10816-10829, 2014.

33. Li Z, Lei H, Luo M, Wang Y, Dong L, Ma Y, Liu C, Song W, Wang F, Zhang J, et al: DNA methylation downregulated mir-10b acts as a tumor suppressor in gastric cancer. Gastric Cancer 18: 43-54, 2015

34. Li Z, Yu X, Wang Y, Shen J, Wu WK, Liang J and Feng F: By downregulating TIAM1 expression, microRNA-329 suppresses gastric cancer invasion and growth. Oncotarget 6: 17559-17569, 2015.

35. Li Z, Yu X, Shen J and Jiang Y: MicroRNA dysregulation in uveal melanoma: A new player enters the game. Oncotarget 6 : 4562-4568, 2015

36. Li J, You T and Jing J: MiR-125b inhibits cell biological progression of Ewing's sarcoma by suppressing the PI3K/Akt signalling pathway. Cell Prolif 47: 152-160, 2014

37. Schirmer U,Doberstein K, Rupp AK, BretzNP, Wuttig D, KiefelH, Breunig C, Fiegl H, Müller-Holzner E, Zeillinger R, et al: Role of miR-34a as a suppressor of L1CAM in endometrial carcinoma. Oncotarget 5: 462-472, 2014

38. Jiang PH, Motoo Y, Garcia S, Iovanna JL, Pébusque MJ and Sawabu N: Down-expression of tumor protein p53-induced nuclear protein 1 in human gastric cancer. World J Gastroenterol 12: 691-696, 2006

39. Tomasini R, Samir AA, Carrier A, Isnardon D, Cecchinelli B, Soddu S, Malissen B, Dagorn JC, Iovanna JL and Dusetti NJ: TP53INP1s and homeodomain-interacting protein kinase-2 (HIPK2) are partners in regulating p53 activity. J Biol Chem 278: 37722-37729, 2003.

40. Liao D, Li T, Ye C, Zeng L, Li H, Pu X, Ding C, He Z and Huang GL: miR-221 inhibits autophagy and targets TP53INP1 in colorectal cancer cells. Exp Ther Med 15: 1712-1717, 2018.

41. Ma S, Tang KH, Chan YP, Lee TK, Kwan PS, Castilho A, Ng I, Man K, Wong N, To KF, et al: miR-130b Promotes CD133(+) liver tumor-initiating cell growth and self-renewal via tumor protein 53-induced nuclear protein 1. Cell Stem Cell 7: 694-707, 2010.

42. Jiang F, Liu T, He Y, Yan Q, Chen X, Wang H and Wan X: MiR-125b promotes proliferation and migration of type II endometrial carcinoma cells through targeting TP53INP1 tumor suppressor in vitro and in vivo. BMC Cancer 11: 425, 2011.

43. Seux M, Peuget S, Montero MP, Siret C, Rigot V, Clerc P, Gigoux V, Pellegrino E, Pouyet L, N'Guessan P, et al: TP53INP1 decreases pancreatic cancer cell migration by regulating SPARC expression. Oncogene 30: 3049-3061, 2011.

44. Giusiano S, Baylot V, Andrieu C, Fazli L, Gleave M, Iovanna JL, Taranger-Charpin C, Garcia S and Rocchi P: TP53INP1 as new therapeutic target in castration-resistant prostate cancer. Prostate 72: 1286-1294, 2012.

45. Gironella M, Seux M, Xie MJ, Cano C, Tomasini R, Gommeaux J, Garcia S, Nowak J, Yeung ML, Jeang KT, et al: Tumor protein 53-induced nuclear protein 1 expression is repressed by miR-155, and its restoration inhibits pancreatic tumor development. Proc Natl Acad Sci USA 104: 16170-16175, 2007.

46. Chaluvally-Raghavan P, Zhang F, Pradeep S, Hamilton MP, Zhao X, Rupaimoole R, Moss T, Lu Y, Yu S, Pecot CV, et al: Copy number gain of hsa-miR-569 at 3q26.2 leads to loss of TP53INP1 and aggressiveness of epithelial cancers. Cancer Cell 26: 863-879, 2014.

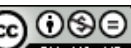

This work is licensed under a Creative Commons Attribution-NonCommercial-NoDerivatives 4.0 International (CC BY-NC-ND 4.0) License. 